\title{
BMJ Open Follow-up study of the regional quota system of Japanese medical schools and prefecture scholarship programmes: a study protocol
}

\author{
Masatoshi Matsumoto, ${ }^{1}$ Keisuke Takeuchi, ${ }^{1}$ Junko Tanaka, ${ }^{2}$ Susumu Tazuma, ${ }^{3}$ \\ Kazuo Inoue, ${ }^{4}$ Tetsuhiro Owaki, ${ }^{5}$ Seitaro Iguchi, ${ }^{6}$ Takahiro Maeda ${ }^{7}$
}

To cite: Matsumoto $\mathrm{M}$, Takeuchi K, Tanaka J, et al. Follow-up study of the regional quota system of Japanese medical schools and prefecture scholarship programmes: a study protocol. BMJ Open 2016;6: e011165. doi:10.1136/ bmjopen-2016-011165

- Prepublication history for this paper is available online. To view these files please visit the journal online (http://dx.doi.org/10.1136/ bmjopen-2016-011165)

Received 15 January 2016 Revised 23 March 2016 Accepted 24 March 2016

CrossMark

For numbered affiliations see end of article.

Correspondence to Dr Masatoshi Matsumoto; matmo10@jb3.so-net.ne.jp

\begin{abstract}
Introduction: Given the shortage of physicians, particularly in rural areas, the Japanese government has rapidly expanded the number of medical school students by adding chiikiwaku (regional quotas) since 2008. Quota entrants now account for $17 \%$ of all medical school entrants. Quota entrants are usually local high school graduates who receive a scholarship from the prefecture government. In exchange, they temporarily practise in that prefecture, including its rural areas, after graduation. Many prefectures also have scholarship programmes for non-quota students in exchange for postgraduate in-prefecture practice. The objective of this cohort study, conducted by the Japanese Council for Community-based Medical Education, is to evaluate the outcomes of the quota admission system and prefecture scholarship programmes nationwide.
\end{abstract}

Methods and analysis: There are 3 groups of study participants: quota without scholarship, quota with scholarship and non-quota with scholarship.

Under the support of government ministries and the Association of Japan Medical Colleges, and participation of all prefectures and medical schools, passing rate of the National Physician License Examination, scholarship buy-out rate, geographic distribution and specialties distribution of each group are analysed. Participants who voluntarily participated are followed by linking their baseline information to data in the government's biennial Physician Census. Results to date have shown that, despite medical schools' concerns about academic quality, the passing rate of the National Physician License Examination in each group was higher than that of all medical school graduates.

Ethics and dissemination: The Ethics Committee for Epidemiological Research of Hiroshima University and the Research Ethics Committee of Nagasaki University Graduate School of Biomedical Sciences permitted this study. No individually identifiable results will be presented in conferences or published in journals. The aggregated results will be reported to concerned government ministries, associations, prefectures and medical schools as data for future policy planning.

\section{Strengths and limitations of this study}

- The regional quota admission system of medica schools is one of the largest national policies ever conducted to redress maldistribution of physicians, and quota entrants now constitute $17 \%$ of all medical school entrants in Japan.

- This study evaluates the outcomes of the quota admission system and prefecture scholarship programmes for medical students by crosssectional survey to prefectures and medical schools, and analysing cohorts of quota and scholarship participants.

- Started in 2013, this is the first comprehensive and longitudinal study of this sort, with support from concerned ministries and the representative body of medical schools.

- All of Japan's prefecture governments and medical schools participate in this study, which yields precise information on the passing rate of the National License Examination for Physicians and scholarship buy-out rate of study participants every year.

- Geographic and specialty distributions of all the study participants voluntarily registered in the cohort can be followed until they retire, as we link the baseline data to the government's national biennial census data, though increasing the recruitment rate of participants is a challenge.

\section{INTRODUCTION}

Geographic maldistribution of physicians in Japan

Geographic maldistribution of physicians has long been recognised as a chronic social problem in Japan. No public agencies have a legal right to decide the allocation of physicians, and thus physicians are given unrestricted freedom to practise wherever they like. There is no allocation system in which local governments or professional bodies place a cap on the number of physicians in 
each area, as seen in other countries such as the UK. In this context, the concentration of physicians in urban areas and a corresponding shortage in rural areas are likely to occur, ${ }^{1-3}$ and initiatives in Japan up to this time have yet to redress the maldistribution of physicians. Article 25 of the Japanese Constitution guarantees healthy living of all citizens and obliges the government to improve public health. So redressing the geographic maldistribution is usually recognised as a responsibility of the national and local governments.

The national and prefectural governments have attempted to resolve this issue in several ways. First, the government constructed one medical school in each prefecture during the 1960s and 1970s, founding new public medical schools in mainly rural prefectures where none had existed. Under this policy, the number of medical schools in Japan increased from 46 to 79 and the number of medical school entrants doubled. ${ }^{4}$ Second, in 1972, the national and prefectural governments cooperated to found a special medical school, Jichi Medical School, solely to train rural physicians. ${ }^{2} 56$ These policies increased both the number of practising physicians nationwide and the number of rural physicians. ${ }^{78}$ It is likely that the increase in the number of rural physicians contributed to the decrease in number of country's designated muichiku (non-physician communities) ${ }^{2}$

However, the equity of distribution of physicians has been almost unchanged; in other words, the disparity between the number of urban and rural physicians has not diminished..$^{7910}$ To make matters worse, a new residency training system was started in 2004. In this new system, similar to the residency matching system of the USA, new graduates are matched to training hospitals nationwide in order of their preferences, which changed the distribution of young physicians who previously tended to concentrate in university hospitals. ${ }^{11}$ This change decreased the physician-assigning capability of university hospitals, which has traditionally dispatched physicians to rural hospitals. ${ }^{3}$ Since the implementation of this new residency scheme, the geographic distribution of physicians has reportedly worsened. ${ }^{12-15}$ The shortage of physicians in rural areas and collapse of healthcare provision in such communities has resurfaced as a social concern.

\section{Emergence of regional quotas as a national policy}

The government responded by rapidly expanding the number of medical school entrants by adding chiikiwaku, a regional quota, in $2008 .{ }^{16}$ Under the quota, applicants to a medical school, in most cases, are required to have graduated from a high school in that prefecture. An expressed intention to practise in that prefecture, preferably in one of its rural areas, is valued in the admission interview. On admission, entrants are usually offered 6-year scholarships from the prefectural government in exchange for working in the prefecture, including its rural areas, for a designated obligation period after

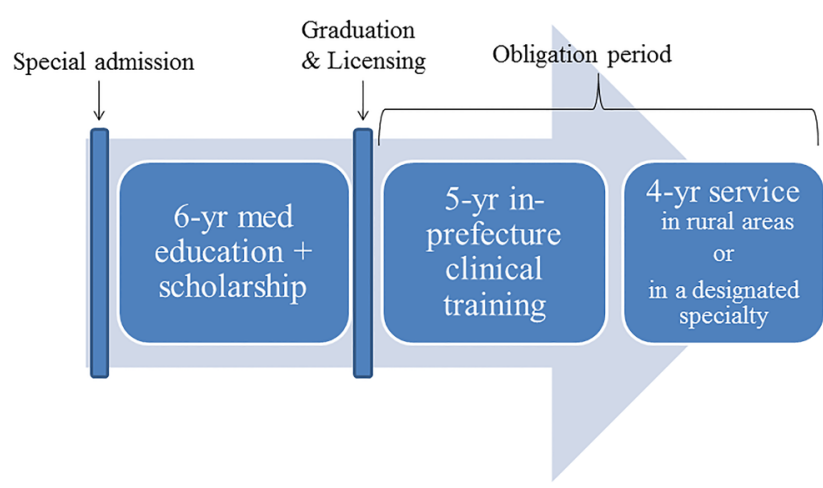

Figure 1 Example of regional quota system of a Japanese medical school (Hiroshima University School of Medicine and Hiroshima Prefecture). Each school designs its own quota system (med, medical; yr, year).

graduation (figure 1). ${ }^{16}$ The design of the quota varies substantially among medical schools. Some medical schools have quotas without scholarship, in which case the intention to practise in certain areas is valued in admission but the entrants have no obligation period. Some quotas require their students to work in rural areas or in a specialty that is recognised to be in severe shortage in the prefecture.

Currently, 70 of Japan's 80 medical schools have such quotas. The number of quota entrants exceeded 1500 per year, amounting to $17 \%$ of all medical school entrants (figure 2). ${ }^{17}$ Historically, this is one of the largest and most ambitious policies to rectify the maldistribution of physicians in Japan and evaluation of outcomes of this policy thus is needed. However, the quota has only just started to graduate students, so there is as yet no comprehensive and longitudinal study to assess its effectiveness.

\section{Prefecture scholarship for medical students independent of quota}

Aside from the quota, many prefectures have independent scholarship programmes for medical students. This scholarship is also given in exchange for postgraduate practice within the prefecture, usually including rural

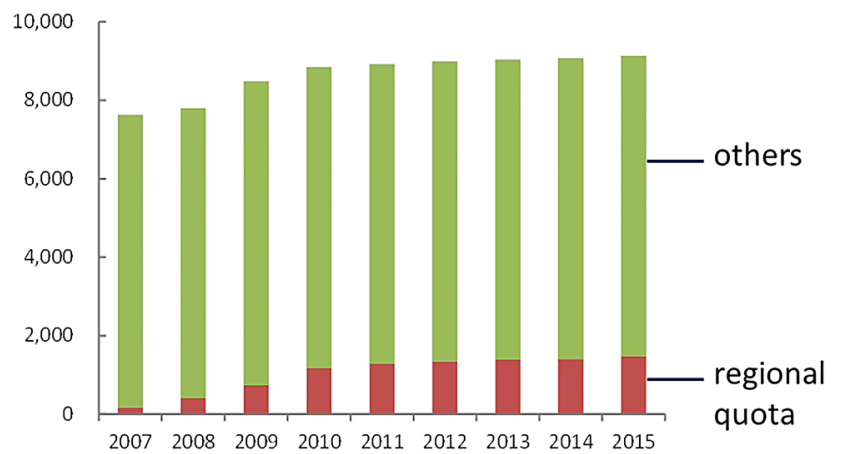

Figure 2 Number of entrants to Japanese medical schools (data from Ministry of Education, Culture, Sports, Science and Technology). ${ }^{17}$ 
areas. The scholarship recipients are students who entered medical schools in the usual way (non-quota admission) or those who entered quotas without having a scholarship. Similar to the quota system, these scholarship programmes have recently expanded. Similar programmes are seen in other countries, but their outcomes are open to debate. ${ }^{18-23}$ The outcomes of the prefecture scholarship programmes in Japan are not yet known.

\section{Objectives of this study}

This cohort study investigates the outcome of the quota admission system of medical schools and that of prefecture scholarship programmes for non-quota students. The cohort consists of three groups of physicians: quota without scholarship, quota with scholarship and scholarship without quota admission. Passing rate of the National Physician License Examination, scholarship buy-out rate, geographic distribution and specialty distribution of each group of participants, are evaluated and compared with those of all Japanese physicians in the same graduating year.

\section{METHODS AND ANALYSIS}

\section{Study design}

This study is conducted by the Japanese Council for Community-based Medical Education (JCCME). The JCCME is the official association of medical school departments engaged in community or rural health education and research. The mission of JCCME is promotion of community-based medical education. Many of the departments in JCCME educate and train quota students.

The outline of study design is shown in figure 3. The study consists of three steps. In the first step, the cohort office of JCCME contacts all prefectural governments and medical schools, and obtains data for the yearly cross-sectional outcomes (lower part of figure 3). In the second step, the cohort office contacts individual participants via prefectural governments or medical schools and obtains baseline data for follow-up (upper part). Finally, in the third step, each individual is followed up by obtaining the corresponding individual's data in the biennial national Physician Census and linking these data to the baseline data.

The Ministry of Health, Labour and Welfare, Ministry of Education, Culture, Sports, Science and Technology, and Association of Japan Medical Colleges support this study by requesting prefectures and/or medical schools to participate in the study.

\section{Definitions of quota and scholarship}

According to this study, a quota of medical schools is one in which 'geographic background or location of graduated high schools of applicants is restricted and/or working place or specialty after graduation is clearly specified'. A scholarship is 'given by a prefecture to a medical student which needs not to be paid back if the student works in designated areas by the prefecture for a certain period'.

In the quota admission system, receiving the scholarship is often a condition for admission to the medical school. However, some medical schools have quotas

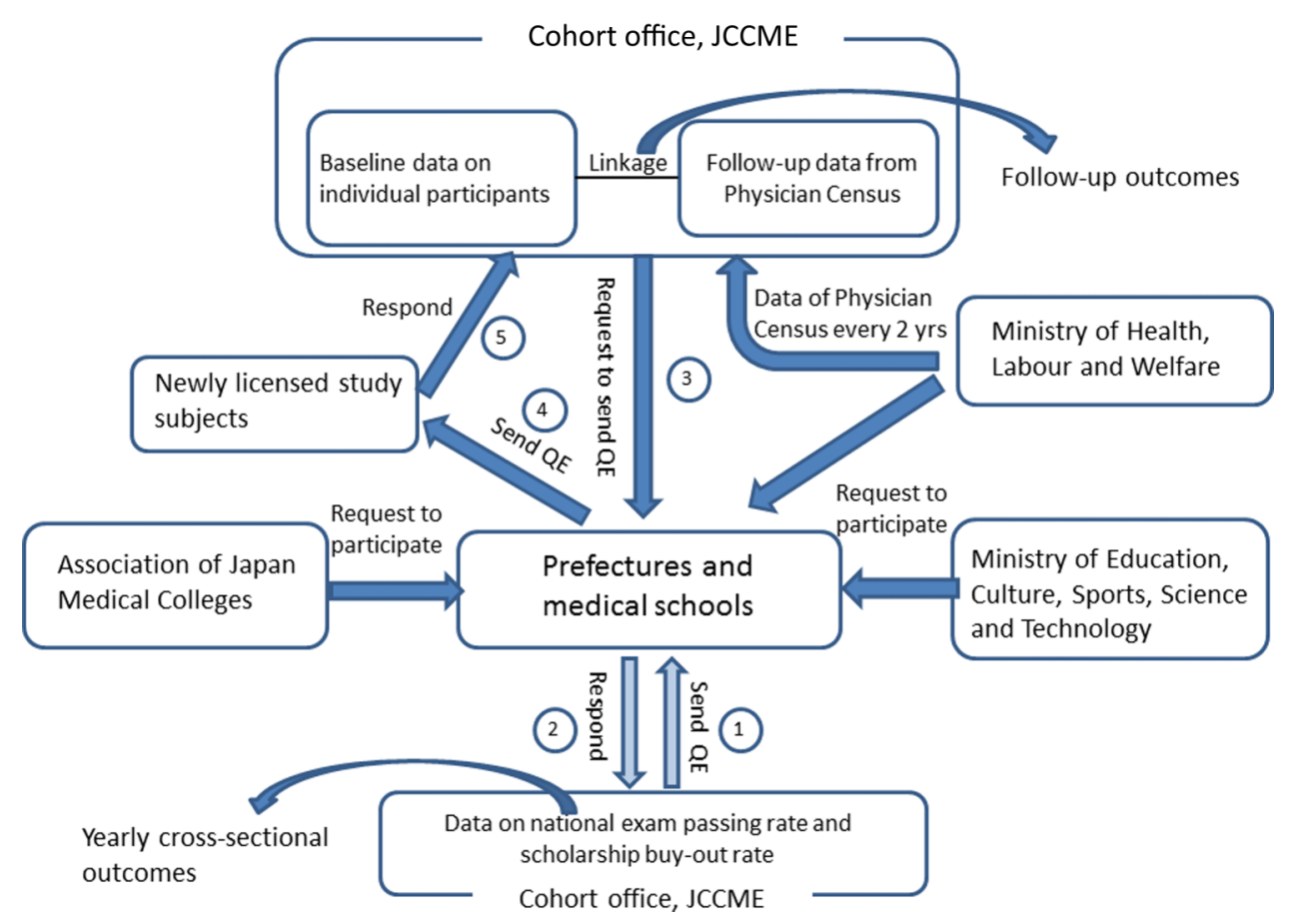

Figure 3 Overview of study design (exam, examination; JCCME, Japanese Council for Community-based Medical Education; $\mathrm{QE}$, questionnaire; yrs, years). 
without scholarships, so entrants are not obligated to practise in certain areas of Japan. In addition, some medical students who did not enter quotas (but did enter medical schools by passing the traditional written examination) may have obtained prefecture scholarships. This study includes participants in all the three groups.

Information on scholarship recipients with or without quota admission is obtained from each prefecture government. Information on participants of quota without scholarship are obtained from each medical school.

\section{Yearly cross-sectional outcomes}

Every year, the cohort office of JCCME begins this study by sending a questionnaire to each prefecture government to obtain information on the number of newly graduated participants who received scholarship, the number of participants who passed the National License Examination for Physicians and the number of participants who bought out the scholarship to be excused from the obligatory work. The cohort office also sends a questionnaire to each medical school to obtain information on the number of participants in quotas without scholarship and the number of participants who passed the National License Examination for Physicians (arrows 1 and 2 in figure 3 ). The results are yearly cross-sectional outcomes. Some results to date are shown later in this paper.

This step of the survey started in 2014 and will be conducted every year at least until 2025.

\section{Individual baseline data}

In the second step, the cohort office requests each prefecture and medical school to forward a questionnaire to each potential study participant (arrow 3 and 4 in figure 3). Each participant then voluntarily sends back the answered questionnaire to the cohort office (arrow $5)$. The cohort office then obtains the baseline data on each participant. The participants are members of one of the three groups (quota alone, quota with scholarship and scholarship alone) and are all newly licensed physicians.

The questionnaire for each participant in the quota group with scholarship and in the scholarship group alone includes information on whether the participant was admitted to a quota, physician identification number, name of the graduated medical school, graduation year, home prefecture, term of scholarship, length of postgraduate obligation period required by the scholarship and length of rural service or service in a designated specialty during the obligation period. The questionnaire for each participant in the quota group without scholarship includes information on physician identification number, name of the graduated medical school, graduation year, home prefecture and conditions for admission to the quota. Demographic data such as age, sex, or marital status, are not included in the questionnaire. The ease in answering the questions was pre-checked using sample participants (quota students) in selected medical schools before the study started. The precision of answers they provided was also assessed by faculty members of the selected schools.

This step in the survey also started in 2014 and will be conducted every year at least until 2025.

\section{Follow-up of individual participants}

The third step is to follow-up the participants. The baseline individual data obtained in the second step are connected, through physician identification number, to the national census data of the 'Survey of Physicians, Dentists and Pharmacists' (Physician Census) conducted by the Ministry of Health, Labour and Welfare, every 2 years (figure 4). All licensed physicians in Japan are obligated to register in the Physician Census. The Physician Census includes information on practice location, type of medical facility, work contents, specialty and board certification status. The connection between the baseline data and the data in Physician Census enables comparison of geographic distribution among the three participant groups, and between each participant group and a comparison group of all physicians in the same graduating year in Japan. The cohort office will request the Ministry of Health, Labour and Welfare to provide individualised data of Physician Census every 2 years beginning in 2016, at which time data of the 2014 Census will be available.

In this way, once the physician identification numbers are stored, the participants can be followed up almost permanently (until the participants retire or stop practising in Japan).

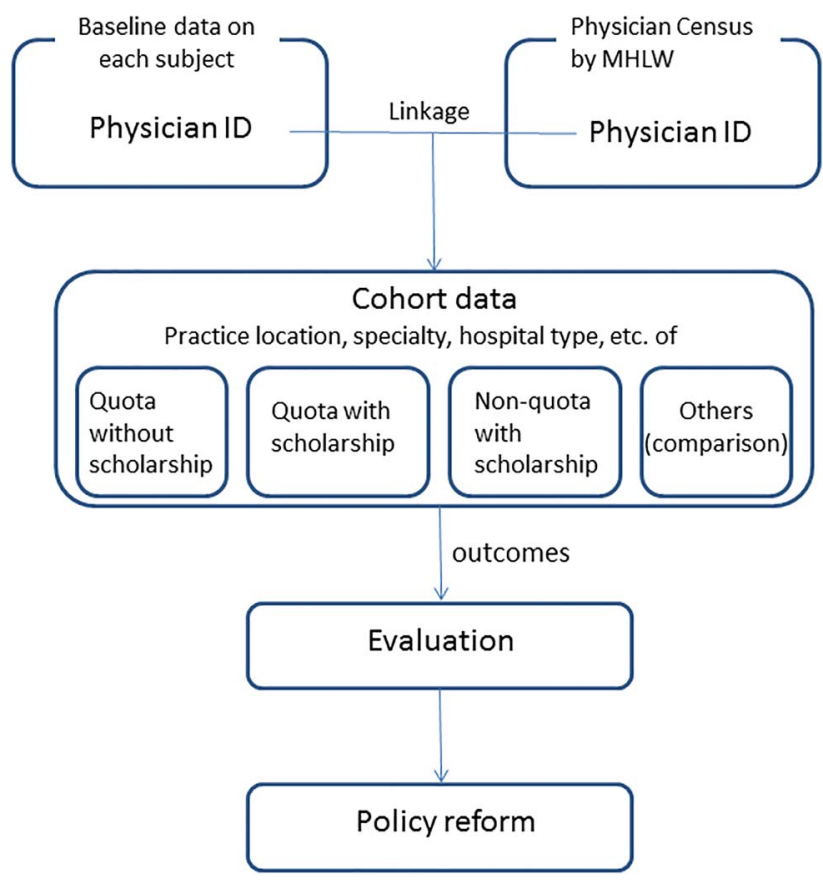

Figure 4 Creation of follow-up data (ID, identification number; MHLW, Ministry of Health, Labour and Welfare). 


\section{Analysis}

On the basis of data obtained directly from the prefectures and medical schools (arrow 2 in figure 3), passing rates of the National License Examination for Physicians in the three groups of participants are calculated. These rates are compared with the passing rates of all medical school graduates in Japan reported each year by the Ministry of Health, Labour and Welfare. Also based on the data from the prefectures, scholarship buy-out rates of participants in the groups of quota with scholarship and scholarship alone are calculated. The buy-out rate is derived from the number of participants who refuse to comply with the conditions accompanied by accepting the scholarship and thus are obliged to return the awarded money.

On the basis of data obtained from individual participants (arrow 5 in figure 3) and their linkage to Physician Census data, municipality (city, town and village)-level distribution, Gini coefficients, proportion of those in rural areas and proportion of those in specialties that are reportedly in shortage nationwide (such as obstetrics and paediatrics) among the participants in each group, are evaluated. These values are compared with those of all the physicians in the same graduating year in the Census (comparison group). The proportion of those who are retained in their home prefectures in each group will be calculated and compared with the proportion of other newly licensed physicians that has been or will be reported elsewhere. ${ }^{17}{ }^{24}$ Also, the associations between baseline characteristics of participants (such as sex and length of obligation period) and their geographic distribution are analysed using multivariate models. These results are longitudinally compared among different postgraduate years in the cohort of the same graduating year.

In short, the results are created based on the data that comprise an annual census of school-level and prefectural-level statistics for the participants, an incomplete census of the cohort participants themselves and a biennial national Physician Census.

\section{RESULTS}

Although follow-up data are yet to be obtained as data of the 2014 Physician Census are still unavailable, crosssectional outcomes in 2014 and 2015 have already been obtained.
In 2014, 38 of Japan's 47 prefectures had study participants who meet the study definitions. All of the 38 prefectures responded to our questionnaire (response rate $100 \%)$. Among all 80 medical schools in Japan, 21 had study participants and all 21 schools $(100 \%)$ responded to our questionnaire. The results of the National License Examination for Physicians among the participants are shown in table 1 . The passing rates of all the three groups of participants were higher than the passing rates of all new medical graduates in Japan: $93.9 \%$.

In 2015, 45 prefectures and 22 medical schools had study participants, and all $(100 \%)$ responded to the questionnaire. As shown in table 1, this year again the passing rates of all the groups were higher than those of all new medical graduates in Japan: $94.5 \% .^{25}$

These results were unexpected, taking into account the concern about academic capability of quota students, a concern that was and still remains among the general population and medical school faculty members.

The number of 2014 licensed participants who bought out their scholarship as of June 2015 was 9 (2.0\%) out of all 446 scholarship awardee participants. The comparable figure of 2015-licensed participants was $4(0.6 \%)$ of the 672 .

As for recruitment of individual participants, in 2014, the cohort office sent a questionnaire, via prefecture or medical school, to 603 participants, and received 241 responses $(40.0 \%)$. In 2015, the questionnaire was sent to 929 participants, and 398 (42.8\%) responded. These respondents were registered as the cohort participants.

\section{Strengths and limitations of this study}

The first strength of this study is that this is the first nationwide and comprehensive longitudinal study on the quota admission system and the prefecture scholarship programme. The second is that, with the help of two related ministries and the national representative body of medical schools, all the prefecture governments and medical schools did and will continue to participate in this study, which enables us to obtain precise information on the number of potential participants, passing rate of the License Examination and scholarship buy-out rate every year. In addition, with the full cooperation of prefectures and medical schools, the cohort office can send the questionnaire to all the potential individual

Table 1 Passing rates of the National License Examination for Physicians

\begin{tabular}{|c|c|c|c|c|c|c|}
\hline & \multicolumn{3}{|l|}{2014} & \multicolumn{3}{|l|}{2015} \\
\hline & $\begin{array}{l}\text { New } \\
\text { graduates }\end{array}$ & Passers & $\begin{array}{l}\text { Passing } \\
\text { rate (\%) }\end{array}$ & $\begin{array}{l}\text { New } \\
\text { graduates }\end{array}$ & Passers & $\begin{array}{l}\text { Passing } \\
\text { rate (\%) }\end{array}$ \\
\hline Quota without scholarship & 166 & 160 & 96.4 & 253 & 244 & 96.4 \\
\hline Quota with scholarship & 144 & 141 & 97.9 & 305 & 295 & 96.7 \\
\hline Non-quota with scholarship & 322 & 305 & 94.7 & 391 & 377 & 96.4 \\
\hline All new graduates in Japan (comparison) & 7749 & 7275 & 93.9 & 8250 & 7798 & 94.5 \\
\hline
\end{tabular}


participants. The third strength of this study is that almost $100 \%$ of participants registered in the cohort can be followed up until they either retire or stop practising in Japan and drop out of the Physician Census. Using the national census data as a way of follow-up costs less money, time and effort, than usual follow-up in which the cohort office would have to contact each potential participant numerous times in the future.

One limitation of this study is that the participation of an individual subject is voluntary and thus recruiting all the potential participants is quite difficult. This is particularly so because the questionnaire includes personal information such as physician identification number. The participation rate of participants in 2014 was $40.0 \%$ and in 2015 it was $43 \%$. We thus cannot exclude a selection bias of the cohort participants, which might create a biased result on the geographic and specialties distribution of the participants in future analyses.

Another limitation is that the baseline data of the participants do not include the content of undergraduate medical education, which makes it impossible to evaluate the effect of undergraduate education, such as rural community-based clinical training, on workforce distribution.

\section{Impact on global, national and local policies}

The special admission systems of medical schools aiming to improve the shortage of physicians in rural areas are conducted worldwide. ${ }^{26-28}$ Also, financial incentive programmes for return of service in underserved areas exist in many countries. ${ }^{22}{ }^{23}$ The results of this study will add evidence to show the effectiveness of these systems and programmes.

The increase in medical school entrants by adding of a quota system will be continued, under a national law, until 2019. But the future of the quota system after 2019 is unknown. The government, prefectures and medical schools have four options: expanding the system, continuing it as it is, restricting its scale and abolishing it entirely. The results of this study, such as geographic distribution, specialty distribution, buy-out rate and national examination passing rate of quota graduates, will enable the stakeholders to make the most informed choice. Moreover, the results of this study in each prefecture will enable the prefecture to consider what to do with its scholarship programme.

\section{DISSEMINATION}

The cohort office explains in a document to all the prefectures, medical schools and individual participants that all the information in the questionnaire is used only for research purposes, and that the data provided by an individual can be deleted at any time by the individual requesting the cohort office to do so. The response to the sent questionnaire is voluntary.

The cohort data are collected and stored in the cohort office (Department of Community-based Medical
System, Hiroshima University) without the personal names of individual participants (completely anonymous data). Aggregated results will be presented in conferences or published in journals. The aggregated results will also be reported to concerned government ministries, associations and prefectures.

\section{Author affiliations}

${ }^{1}$ Department of Community-Based Medical System, Institute of Biomedical and Health Sciences, Hiroshima University, Hiroshima, Japan

${ }^{2}$ Department of Epidemiology, Infectious Disease Control and Prevention, Institute of Biomedical and Health Sciences, Hiroshima University, Hiroshima, Japan

${ }^{3}$ Department of General Internal Medicine, Hiroshima University Hospital and Graduate School of Biomedical \& Health Sciences, Hiroshima, Japan

${ }^{4}$ Department of Community Medicine, Chiba Medical Center, Teikyo University School of Medicine, Chiba, Japan

${ }^{5}$ Education Center for Doctors in Remote Islands and Rural Areas, Graduate School of Medical Sciences, Kagoshima University, Kagoshima, Japan ${ }^{6}$ Department of Community Medicine, Niigata University Graduate School of Medical \& Dental Sciences, Niigata, Japan

${ }^{7}$ Department of Community Medicine, Nagasaki University Graduate School of Biomedical Science, Nagasaki, Japan

Contributors MM contributed to the study design, tools, study administration, data collection, analysis and interpretation of data and writing of the draft. KT and TM contributed to the study design, tools, study administration, data interpretation and writing of the draft. JT, ST and KI contributed to the study design, interpretation of data and writing of the draft. TO and SI contributed to the study administration, data collection and writing of the draft.

Funding This study is funded by the Ministry of Education, Culture, Sports, Science and Technology KAKENHI Grant-in-Aid for Scientific Research (C), grant number (25460803).

\section{Competing interests None declared}

Ethics approval Ethical approval was granted by the Ethics Committee for Epidemiological Research of Hiroshima University (reference number 778) and the Research Ethics Committee of Nagasaki University Graduate School of Biomedical Sciences (reference number 13091342).

Provenance and peer review Not commissioned; externally peer reviewed.

Data sharing statement Conditions of the ethical approvals permit the cohort office (Department of Community-Based Medical System, Institute of Biomedical and Health Sciences, Hiroshima University) and the suboffice (Department of Community Medicine, Nagasaki University Graduate School of Biomedical Science) to share the cohort data. Aggregated data are shared with stakeholders or other researchers.

Open Access This is an Open Access article distributed in accordance with the Creative Commons Attribution Non Commercial (CC BY-NC 4.0) license, which permits others to distribute, remix, adapt, build upon this work noncommercially, and license their derivative works on different terms, provided the original work is properly cited and the use is non-commercial. See: http:// creativecommons.org/licenses/by-nc/4.0/

\section{REFERENCES}

1. Matsumoto M, Inoue K, Farmer J, et al. Geographic distribution of primary care physicians in Japan and Britain. Health Place 2010;16:164-6.

2. Matsumoto $\mathrm{M}$, Inoue $\mathrm{K}, \mathrm{Kajii} \mathrm{E}$, et al. Retention of physicians in rural Japan: concerted efforts of the government, prefectures, municipalities and medical schools. Rural Remote Health 2010;10:1432

3. Ikegami N. Universal health coverage for inclusive and sustainable development: lessons from Japan. The World Bank, Washington DC 2014.

4. Asano N, Kobayashi Y, Kano K. Issues of intervention aimed at preventing prospective surplus of physicians in Japan. Med Educ $2001 ; 35: 488-94$. 
5. Inoue K, Hirayama Y, Igarashi M. A medical school for rural areas. Med Educ 1997;31:430-4.

6. Matsumoto M, Inoue K, Kajii E. Contract-based training system for rural physicians: follow-up of Jichi Medical University graduates (1978-2006). J Rural Health 2008;24:360-8.

7. Inoue $\mathrm{K}$, Matsumoto $\mathrm{M}$, Toyokawa $\mathrm{S}$, et al. Transition of physician distribution (1980-2002) in Japan and factors predicting future rural practice. Rural Remote Health 2009;9:1070.

8. Matsumoto $\mathrm{M}$, Inoue $\mathrm{K}$, Bowman $\mathrm{R}$, et al. Physician scarcity is a predictor of further scarcity in US, and a predictor of concentration in Japan. Health Policy 2010;95:129-36.

9. Kobayashi Y, Takaki H. Geographic distribution of physicians in Japan. Lancet 1992;340:1391-3.

10. Matsumoto M, Inoue K, Bowman R, et al. Geographical distributions of physicians in Japan and US: impact of healthcare system on physician dispersal pattern. Health Policy 2010;96:255-61.

11. Koike $\mathrm{S}$, Ide $\mathrm{H}$, Yasunaga $\mathrm{H}$, et al. Postgraduate training and career choices: an analysis of The National Physicians Survey in Japan. Med Educ 2010;44:289-97.

12. Toyabe S. Trend in geographic distribution of physicians in Japan. Int J Equity Health 2009;8:5.

13. Tanihara $\mathrm{S}$, Kobayashi $\mathrm{Y}$, Une $\mathrm{H}$, et al. Urbanization and physician maldistribution: a longitudinal study in Japan. BMC Health Serv Res 2011;11:260.

14. Sakai R, Wang W, Yamaguchi N, et al. The impact of Japan's 2004 postgraduate training program on intra-prefectural distribution of pediatricians in Japan. PLos ONE 2013;8:e77045.

15. Sakai R, Tamura H, Goto R, et al. Evaluating the effect of Japan's 2004 postgraduate training programme on the spatial distribution of physicians. Hum Resour Health 2015;13:5.

16. Matsumoto M, Inoue K, Takeuchi K. Quality of care in Japan: an additional strategy. Lancet 2011;378:e17.

17. Ministry of Education, Culture, Sports, Science and Technology. Survey on community health. http://www.mext.go.jp/component/
a_menu/education/detail/__icsFiles/afieldfile/2015/11/17/1324090_ 19.pdf

18. Rosenblatt RA, Saunders G, Shreffler J, et al. Beyond retention: National Health Service Corps participation and subsequent practice locations of a cohort of rural family physicians. J Am Board Fam Pract 1996;9:23-30

19. Pathman DE, Konrad TR, Ricketts TC III. The comparative retention of National Health Service Corps and other rural physicians. Results of a 9-year follow-up study. JAMA 1992;268:1552-8.

20. Pathman DE, Fryer GE Jr, Phillips RL, et al. National Health Service Corps staffing and the growth of the local rural non-NHSC primary care physician workforce. J Rural Health 2006;22:285-93.

21. Anderson M, Rosenberg MW. Ontario's underserviced area program revisited: an indirect analysis. Soc Sci Med 1990;30:35-44.

22. Bärnighausen T, Bloom DE. Designing financial-incentive programmes for return of medical service in underserved areas: seven management functions. Hum Resour Health 2009;7:52.

23. Bärnighausen T, Bloom DE. Financial incentives for return of service in underserved areas: a systematic review. BMC Health Serv Res 2009;9:86.

24. Ministry of Health, Labour and Welfare. Geographic movement of young physicians. http://www.mhlw.go.jp/stf/shingi/2r985200000 32oxz-att/2r98520000032p1w_1.pdf

25. Ministry of Health, Labour and Welfare. Results of the 109th National License Examination for Physicians. http://www.mhlw.go.jp/general/ sikaku/successlist/siken01/about.html

26. Rabinowitz HK. Recruitment, retention, and follow-up of graduates of a program to increase the number of family physicians in rural and underserved areas. N Engl J Med 1993;328:934-9.

27. Sriratanaban A, Sangprasert B. The Rural Area Project (RAP) in Thailand: curriculum development. Med Educ 1983;17:374-7.

28. Rabinowitz HK, Paynter NP. The role of the medical school in rural graduate medical education: pipeline or control valve? J Rural Health 2000;16:249-53. 